\title{
HIV Pre-Exposure Prophylaxis (PrEP) Knowledge, Familiarity, and Attitudes among Healthcare Professional Students
}

\section{Sarahmona Przybyla ( $\sim$ mona@buffalo.edu )}

University at Buffalo - The State University of New York https://orcid.org/0000-0001-9283-7307 Jennifer Fillo

University at Buffalo - The State University of New York

Kimberly Kamper-DeMarco

SUNY College at Buffalo

\section{Kathleen Parks}

University at Buffalo - The State University of New York

Jacob Bleasdale

University at Buffalo - The State University of New York

Lynne Klasko-Foster

Brown University

\section{Diane Morse}

University of Rochester Eastman School of Music Official Bookstore

\section{Research article}

Keywords: Healthcare Professional education, Students, PrEP, HIV prevention, Education, Health Personnel

Posted Date: July 31st, 2019

DOI: https://doi.org/10.21203/rs.2.12214/v1

License: (a) (1) This work is licensed under a Creative Commons Attribution 4.0 International License. Read Full License 


\section{Abstract}

Background The federal government's plan to End the HIV Epidemic by 2030 includes a primary goal to reduce new HIV infections by 90 percent. One key contributor to this plan is pre-exposure prophylaxis (PrEP), a medication taken by HIV-uninfected individuals to prevent infection. While knowledge and acceptance of PrEP among clinicians is growing, few studies have assessed knowledge and awareness among future healthcare professionals in academic training programs. Given the limited research on PrEP among healthcare professional students, a better understanding of knowledge and familiarity with PrEP prescription may inform the development of educational interventions targeting this population in an effort to increase implementation. The present study aimed to assess and compare healthcare trainees' PrEP awareness, knowledge, and familiarity with prescription guidelines with a broad goal to better understand and prevent gaps in academic training regarding PrEP. Methods A cross-sectional webbased survey of medical, nurse practitioner, and pharmacy students enrolled at two universities was conducted between October 2017-January 2018. The study assessed participants' awareness, knowledge, and familiarity with PrEP prescribing guidelines and willingness to prescribe PrEP and refer to another healthcare provider. Results The survey was completed by 744 participants (overall response rate $=36.2 \%$ ). There were significant differences among student groups in all domains. Pharmacy students had the greatest PrEP knowledge, awareness, and familiarity with prescribing guidelines. However, medical students reported the greatest comfort with performing PrEP-related clinical activities and willingness to refer a candidate to another provider. Greater familiarity with PrEP prescription guidelines was associated with willingness to prescribe the medication. Conclusions Healthcare professional students' responses enhance our understanding of the relevant experiences and perspectives of future healthcare providers that influence their knowledge towards the promotion of PrEP through prescription and referral intentions. The gaps in students' knowledge offer opportunities for the development of educational strategies to support HIV prevention among future healthcare professionals.

\section{Background}

Clinical trials have repeatedly demonstrated the effectiveness of daily oral pre-exposure prophylaxis (PrEP) (1-3) for its HIV preventive use among at-risk adults, leading to the Food and Drug Administration (FDA)'s 2012 approval of Truvada ${ }^{\circledR}$ (tenofovir disoproxil fumerate/emtricitabine TDF/FTC). Two years later, the Centers for Disease Control and Prevention (CDC) issued PrEP clinical practice guidelines for selecting, prescribing, and following eligible patients (4). In 2018, the FDA approved TDF/ FTC for use in adults and adolescents who weigh at least 35 kilograms. PrEP provides a significant public health opportunity to decrease HIV incidence. Indeed, the uptake of PrEP in the United States is associated with decreases in HIV diagnoses (5). However, uptake has not kept pace with original expectations; estimates indicate that 269,000 people are on PrEP (6), despite more than one million Americans meeting eligibility guidelines (7). Although PrEP adoption has grown, coverage is not evenly distributed across gender or geography (8). The federal government's plan to End the HIV Epidemic by 2030 includes a primary goal to reduce new HIV infections by 90 percent. The opportunity for PrEP to make a meaningful impact on HIV 
incidence is dependent upon not only large-scale adoption among eligible populations, but also the identification of gaps in awareness and knowledge among healthcare providers that may influence prescription and referral behaviors. This second point warrants attention as clinician-directed efforts to increase PrEP uptake need to consider provider type and training experiences that will lead to greater PrEP implementation (9).

One of the understated triumphs of PrEP revolves around general awareness among healthcare providers. Since FDA approval, overall PrEP awareness has grown with four recent surveys of clinicians demonstrating awareness between $64 \%-93 \%$ (10-13). In addition, clinician willingness to prescribe PrEP has increased, particularly among infectious disease specialists and HIV care providers relative to primary care providers (14-19). However, a key concern is that the growth in awareness has not corresponded with an increase in the number of providers who prescribe the medication. Importantly, knowledge translates to behavior; PrEP knowledge is consistently associated with actual prescribing practices (20-22).

To date, there is limited data about healthcare professional students' knowledge and perceptions of PrEP as future potential prescribers. The few studies were limited to medical students (23-26) and did not include other health professional students who may play an active role in PrEP care, such as those in nurse practitioner, or pharmacy training programs. Calabrese and colleagues surveyed medical students regarding the influence of patient race (23), sexual orientation (24), and sexual behavior (25) on clinical decision-making related to PrEP. Results demonstrate that medical students' awareness of PrEP was generally high (85\%), with half reporting PrEP education during medical school. In another small study with 112 medical students, half of the sample reported some PrEP knowledge and approximately three quarters reported willingness to recommend PrEP to patients (26). A recent editorial written by medical students articulated their desire for widespread PrEP implementation as a key preventive element to reduce HIV infections (27). Together, findings from empirical studies coupled with stated medical students' commentary point towards the need for greater knowledge of PrEP and prescribing and referral intentions for at-risk populations.

Given the current state of the literature on PrEP among healthcare providers, the timing is now ideal to shift the conversation to clinician training experiences for the next generation of healthcare providers. If the potential for PrEP efficacy is to be maximized, public health and medical researchers recognize that knowledge, training, and engagement must be accelerated in groups who serve as gatekeepers to PrEP, including healthcare professionals in a variety of disciplines, not limited to physicians or specifically infectious disease specialists. Given the limited evidence on future healthcare professionals' awareness of PrEP, a better understanding of knowledge and familiarity with PrEP prescription may inform the development of educational interventions targeting this population in an effort to increase implementation. The present study aimed to assess healthcare trainees' PrEP awareness, knowledge, familiarity with prescribing guidelines, and willingness to prescribe and refer to another provider with a broad goal to better understand and prevent gaps in academic PrEP training. 


\section{Methods}

\section{Study Participants and Procedures}

Students currently enrolled in nurse practitioner (NP), pharmacy (PharmD), and medical (MD) programs at two universities in New York State were eligible to participate in a web-based cross-sectional survey that aimed to examine knowledge and perceptions towards PrEP. Between October 2017-January 2018, potential participants at one university $(n=1,404)$ received an email from the principal investigator $(\mathrm{PI})$ delivered to their university-affiliated email address inviting them to participate. The second university sent emails directly to students $(n=649)$ from their respective Dean inviting them to contact the PI if they were interested in participating. All emails contained a cover letter explaining study purpose and procedures. A unique link was embedded in the email invitation to access the survey, which prevented multiple completions per person. Those individuals willing to participate in the study continued by answering the survey questions to indicate consent to participate. Non-responders were sent weekly requests to complete the survey with a maximum of three reminder emails. The overall response rate was $36.2 \%$. Survey length averaged 15.4 minutes, and data were stored in a password-protected data file unlinked to participants' identifying information. Participants received a \$10 electronic Amazon gift card. The study protocol was approved by the universities' institutional review boards with a waiver of written informed consent. All procedures performed were in accordance with the ethical standards of the institutional research committees and with the 1964 Helsinki declaration and its later amendments or comparable ethical standards.

\section{Measures}

Participant background characteristics were collected including age, race, sex, academic training program (i.e., MD, NP, PharmD) and year in current program. Survey items were developed using previous studies among clinicians as a template $(12,14)$. PrEP Awareness was determined by a yes $(1) / \mathrm{no}(0)$ response to the question: "Prior to this survey, were you aware of the concept of HIV PrEP (pre-exposure prophylaxis), which is prescribing regularly-scheduled oral HIV medications to HIV-uninfected individuals to help prevent them from getting HIV?" Familiarity with PrEP Prescription was assessed with one item that asked, "Please rate how familiar or unfamiliar you consider yourself with regard to prescribing PrEP" on a Likert scale ranging from (1) very unfamiliar to (5) very familiar. PrEP Knowledge was measured with six multiple-choice items based on CDC's PrEP clinical guidelines with responses scored as correct (1) or incorrect (0). Scores were summed across items to create a total knowledge score. These items included knowledge of PrEP dosing frequency, approved FDA medications, HIV antibody testing prior to PrEP initiation, HIV testing frequency while on PrEP, contraindications to prescribing PrEP, and routine standard of care practices for patients taking PrEP. Those participants who responded with no familiarity with prescribing guidelines were assumed to lack knowledge and therefore were coded as zero on the knowledge items. Comfort Performing PrEP-Related Clinical Activities was assessed with three items as relevant foundational tasks to the provision of PrEP (e.g., discussing sexual activities) (14). Items were rated on a Likert scale ranging from (1) completely uncomfortable to (5) completely comfortable, and 
scores were averaged across items. The primary dependent variables of interest included respondents' willingness to prescribe PrEP and willingness to refer a PrEP candidate to another healthcare provider, both of which were measured on a Likert scale ranging from (1) completely unwilling to (5) completely willing.

\section{Data Analytic Plan}

Data were analyzed in three stages. First, descriptive analyses were conducted to characterize the sample. Second, analysis of variance (ANOVA) was used to compare key variables (i.e., PrEP awareness, PrEP knowledge, familiarity with prescribing guidelines, comfort with performing PrEP-related clinical activities, experience with HIV-infected populations) across the three student groups. Finally, linear regression models were conducted to compare differences across groups in: a) willingness to prescribe PrEP, and b) willingness to refer a PrEP candidate to another provider. In the former model, comparisons were made between MD (1) and NP (0) students, given that PharmD students are unable to prescribe PrEP in most states. In the latter models, comparisons were made across student groups. Models controlled for participant sex, race, program year, familiarity with PrEP prescription guidelines, PrEP knowledge, and knowing someone who has used PrEP. All analyses were conducted using IBM SPSS Statistics (Version 25).

\section{Results}

\section{Preliminary Analyses}

Participant characteristics for the full sample and by student type are presented in Table 1. The analytic sample was composed of 744 participants: Participants were primarily female (61.2\%) and White (65.8\%) or Asian $(24.2 \%)$ with a mean age of 25.1 years $(S D=4.25)$. Descriptive statistics on participants' prior exposure to HIV-infected populations and knowledge of PrEP users are presented at the bottom of Table 1. The majority $(65.02 \%)$ had experience with HIV-infected populations, but this varied by group $(F(2,741)$ $=54.129, p<.001)$; NP students had the most experience (92.4\%), followed by MD students $(69.9 \%)$ and PharmD students (40.8\%). The majority of participants did not know someone who has used PrEP $(72.5 \%)$, and there were no differences across groups $(F(2,736)=0.170, p=.844)$.

\section{Focal Analyses}

Results of ANOVAs comparing student groups on key variables are presented in Table 2. There were significant group differences in PrEP awareness $[F(2,741)=4.42, p=.012]$, PrEP knowledge $[F(2,738)=11.0, p<.001]$, familiarity with prescription guidelines $[F(2,739)=41.61, p<.001]$, and comfort with performing PrEP-related clinical activities $[\mathrm{F}(2,739)=3.64, p=.027]$. PharmD students had greater PrEP awareness than NP students $(t=-2.87, p=.004)$. PharmD students had greater PrEP knowledge than both MD students $(t=-4.67, p<.001)$ and NP students $(t=-2.10, p=.036)$. PharmD students had 
greater familiarity with prescription guidelines than both MD students $(t=-8.19, p<.001)$ and NP students $(t=-6.62, p<.001)$. However, MD students were more comfortable with performing PrEP-related clinical activities than PharmD students $(t=2.45, p=.014)$.

In the first regression model, we examined differences between MD and NP students in willingness to prescribe PrEP (see Table 3). Analyses controlled for participant sex, race, program year, and familiarity with PrEP prescribing guidelines, PrEP knowledge, and whether participants know a person who has used PrEP. Results revealed a non-significant trend suggesting that MD students may be more willing to prescribe PrEP in their clinical practice $(b=0.19, p=.061)$. There were also significant main effects of program year $(b=-0.10, p=.004)$ and familiarity with PrEP prescription guidelines $(b=0.09, p=.003)$. Willingness to prescribe PrEP was lower among participants further along in their respective programs. Additionally, greater familiarity with PrEP prescription guidelines was associated with greater willingness to prescribe PrEP.

In subsequent regression models, we examined group differences in willingness to refer a PrEP candidate to another provider (see Table 4). Results revealed a significant main effect of student type indicating that MD students were more willing to refer a PrEP candidate to another provider than were PharmD students $(b=0.11, p=.037)$. However, there were no differences between NP and PharmD students $(b=$ $.05, p=.563)$, nor between MD and NP students $(b=0.06, p=.429)$. There were no significant main effects for any of the covariates in these models.

\section{Discussion}

PrEP uptake is dependent on healthcare provider awareness, knowledge of eligibility criteria, and ability to either prescribe or make an appropriate referral. The purpose of this investigation was to assess awareness, knowledge, and familiarity with PrEP guidelines and their relation to PrEP prescription and referral intentions among healthcare professional students representing three different disciplines. This is the first study to examine a large sample of clinicians in academic training programs who have the capacity to become part of the PrEP prescription or referral process. Findings indicate that PrEP awareness, knowledge, and familiarity with prescribing guidelines differs by discipline, with PharmD students reporting the highest levels of each. Medical students reported the highest comfort prescribing PrEP, greater familiarity with PrEP and increased willingness to prescribe. These results enhance our understanding of the relevant experiences and perspectives of future healthcare providers that influence their knowledge towards the promotion of PrEP.

\section{PrEP Care Continuum}


Utilizing PrEP as an HIV prevention tool is a more complex process than simply prescribing the drug, patient monitoring, and prescription renewal. The PrEP care continuum is a multi-step process that necessitates intervention focused on PrEP awareness, uptake, and adherence and retention (28). Pharmacists, nurse practitioners, and physicians play key roles in each of these steps. Understanding gaps in training can inform professional education to increase comfort and willingness to intervene across the PrEP care continuum as students transition into their careers.

\section{Interdisciplinary Approaches to PrEP Care}

In this sample, PharmD students had the greatest PrEP awareness, PrEP knowledge, and familiarity with prescribing guidelines. They also reported the lowest exposure to HIV-infected patient populations. Taken together, this suggests that PharmD programs may be providing more PrEP-related education than other practical healthcare disciplines. Community pharmacists are traditionally utilized in PrEP care through filling prescriptions and counselling patients taking PrEP as part of the adherence and retention step of the PrEP care continuum. While pharmacists do not have prescribing privileges in New York State, they are uniquely positioned to educate community members about HIV prevention options including PrEP, and have the ability to refer PrEP candidates to providers, thereby becoming involved in both the awareness and uptake steps in PrEP care. Compared to students in other disciplines, PharmD students were less willing to refer a potential PrEP candidate. As such, emphasis on reducing barriers to referrals during academic training may be an important intervention to encourage pharmacists to view themselves as significant contributors in PrEP care beyond maintenance stages. Furthermore, pharmacists may be more integral to adherence and retention than prescription refills alone due to factors such as advanced knowledge, geographic accessibility to vulnerable populations, and patient trust and rapport (29). Similar to their role in HIV treatment maintenance, pharmacists have unique access to data on timeliness of PrEP refill as well as periodic patient interactions $(30,31)$. As such, delayed refills could prompt pharmacists to discuss PrEP adherence with patients, building relationships to reinforce and optimize PrEP retention. More focus on PrEP counselling is needed during graduate training to increase confidence in patient education across the PrEP care continuum (32).

Nurse practitioner students reported lower PrEP awareness, PrEP knowledge, familiarity with prescribing guidelines, and relatedly, less comfort prescribing PrEP than students in other disciplines. This is a disappointing finding as the great majority of NP students in the sample had at least some experience with HIV-infected patient populations and many of them may continue to interface with vulnerable populations at increased risk for HIV acquisition. Previous work has shown prior exposure to HIV-infected populations to be a strong predictor of intentions to prescribe $\operatorname{PrEP}(12,15)$. Additionally, nurse practitioners have a skillset that can be uniquely leveraged to decrease PrEP barriers and increase coverage in high-risk populations. As outlined by Nelson and colleagues (33), nurse-led approaches across the PrEP care continuum are a natural fit based on discipline-related assets including holistic approaches to patient care (e.g., patient education to increase awareness of HIV risk), advanced clinical skills to support PrEP uptake, and the ability to translate new evidence into practice to support adherence and retention. Because NPs are likely to encounter PrEP candidates, increasing their awareness and 
knowledge of PrEP and providing training on prescription guidelines is a vital step to increasing selfefficacy to prescribe, and subsequently increasing PrEP prescription coverage. As PharmD students reported increased PrEP knowledge, awareness, and familiarity with prescribing guidelines, there may be opportunities for transdisciplinary training, whereby NP students could attend existing workshops or seminars with PharmD students. This collaborative training may present a unique opportunity for introducing referral skills, and students could benefit from such an interdisciplinary approach. Furthermore, doctoral nursing education already includes competencies that align with the PrEP care continuum. Educational modules could include reframing such competencies within the context of PrEP.

Despite their lower PrEP awareness, PrEP knowledge, and familiarity with prescribing guidelines, MD students were most willing to refer and marginally more likely to prescribe PrEP. While uptake and adherence are natural intervention points for physicians within the context of PrEP care, their important position in awareness-raising and linkage to care should not be discounted. Perhaps lower knowledge and familiarity with prescribing contributes to the desire to refer patients to a more knowledgeable provider. In the current sample, program year was inversely related to willingness to prescribe, which may be attributable to deciding on a specialty in later program years (e.g., dermatology) that is less intuitively related to PrEP prescription. Increasing baseline knowledge to raise PrEP awareness and confidence to discuss sexual risk with patients regardless of specialty, paired with ability to refer to appropriate PrEP providers has the capacity to increase PrEP coverage at the population level. However, optimal uptake is still dependent on maximizing the number of potential prescribers. Increasing one's ability to prescribe PrEP and retain patients in preventive care for medical students embarking on careers beyond infectious disease (e.g., primary care, family medicine, pediatrics) is an important target for intervention with physicians, especially since familiarity with PrEP prescribing guidelines is a significant predictor of willingness to prescribe. Additionally, graduate medical courses should work to debunk racial and other stereotypes about risk compensation, as such biases impede provider willingness to prescribe PrEP (23$25,34)$. Future work could examine the extent to which hands-on experiences with high-risk populations (e.g., via clinical rotations) impact both willingness to prescribe and refer.

\section{Implications}

While New York has the fourth highest HIV incidence in the nation, the state has vowed to end the AIDS epidemic and is committed to increasing PrEP coverage as part of the strategy. Academic training programs in medicine, nursing, and pharmacy are key points of intervention to help achieve this goal. The 16 public and private medical schools in the State of New York train approximately $11 \%$ of the country's medical students, nearly half of whom remain in the State to practice medicine. In 2017, New York State medical school enrolment was 10,743, an increase of more than $25 \%$ from 2002 (35). As key stakeholders in PrEP utilization in the future, medical students have an important role to play as future healthcare providers. In addition, New York houses 11 accredited DNP programs and 8 accredited PharmD programs, licensing over 2,500 nurse practitioners and 1,300 doctors of pharmacy over the last 5 years (36-39). Engaging with current students to better understand their PrEP knowledge in the future is critical in advancing the research field as prescribing patterns continue to grow in a variety of healthcare 
practice settings (40). Importantly, increasing knowledge and acceptance of PrEP via educational training of healthcare professionals has the potential to be both cost effective and to broadly influence the provision of PrEP to those at increased risk for HIV infection (41).

Findings of this work have implications for identifying potential intervention points in the training of students in healthcare professional programs to increase their capacity to prescribe directly or refer to other providers. These results echo recommendations in other work suggesting the need to expand healthcare professional students' training on PrEP (26). As the PrEP continuum of care involves intervention points at patient awareness, uptake, and adherence and retention, interdisciplinary workforce training may be key to improving coverage. Case-based learning to facilitate active learning has been used in other medical school curricula when covering infectious disease content $(42,43)$ and may be a model for other health professional training programs to use when covering PrEP. This type of experiential learning may be ideal for students to work synergistically across disciplines and leverage knowledge already present in independent curricula. Importantly, the primary goal of this novel study is to identify gaps in knowledge to aid in development of educational programs to improve awareness, attitudes, comfort, and willingness to adopt PrEP among future healthcare professionals. While it is optimistic to expect growing PrEP uptake, more research is needed to understand future healthcare professionals' knowledge of PrEP and the transition to actual prescription or referral behaviors.

\section{Limitations}

Given that this convenience sample was recruited from two universities in one state, these results are not generalizable to health professional students broadly speaking. Our results also may be affected by nonresponse bias as students who had no knowledge of PrEP may have chosen not to participate. Despite these limitations, this study provides one of the first examinations of PrEP knowledge and perceptions among future healthcare professionals.

\section{Conclusions}

The current study examined PrEP awareness, knowledge, and familiarity with PrEP guidelines and their relation to PrEP prescription and referral intentions among healthcare professional students representing three different disciplines. As a wide variety of healthcare providers and support staff are essential to the provision of PrEP in the community, it is vital that academic training programs provide resources for future healthcare professionals to provide PrEP to clinically eligible PrEP candidates. Increasing PrEP coverage in the United States involves healthcare providers across disciplines; consequently, academic education efforts should increase opportunities for interdisciplinary training.

\section{Abbreviations}

CDC: Centers for Disease Control and Prevention

DNP: Doctor of Nursing Practice 
FDA: Food and Drug Administration

MD: Doctor of Medicine

NP: Nurse Practitioner

PharmD: Doctor of Pharmacy

PrEP: Pre-exposure Prophylaxis

\section{Declarations}

Ethics approval and consent to participate

The study protocol was approved under exempt status by the universities' institutional review boards. All procedures performed were in accordance with the ethical standards of the institutional research committees and with the 1964 Helsinki declaration and its later amendments or comparable ethical standards.

\section{Consent for publication}

Not applicable.

\section{Availability of data and material}

The datasets used and/or analyzed during the current study are available from the corresponding author on reasonable request

\section{Competing interests}

The authors declare that they have no competing interests.

\section{Funding}

Funding for this research was supported in part by the University of Rochester Center for AIDS Research grant P30AI078498 (NIH/NIAID) and the University of Rochester School of Medicine and Dentistry through a grant awarded to SP and DM. Preparation of this manuscript was partially supported by NIAAA Grant T32AA007583 in support of JF and KK. 
Author's contributions

SP, KP and DM designed the research study. SP, DM, KP and JB performed the research. JF and KK analyzed the data. All authors contributed to the writing of the paper.

\section{Acknowledgements}

The authors wish to thank the students who generously contributed their time and effort by participating in this study.

\section{References}

1. Baeten JM, Donnell D, Ndase P, Mugo NR, Campbell JD, Wangisi J, et al. Antiretroviral prophylaxis for HIV prevention in heterosexual men and women. N Engl J Med. 2012;367(5):399-410.

2. Grant RM, Lama JR, Anderson PL, McMahan V, Liu AY, Vargas L, et al. Preexposure chemoprophylaxis for HIV prevention in men who have sex with men. N Engl J Med. 2010;363(27):2587-99.

3. Thigpen MC, Kebaabetswe PM, Paxton LA, Smith DK, Rose CE, Segolodi TM, et al. Antiretroviral preexposure prophylaxis for heterosexual HIV transmission in Botswana. N Engl J Med. 2012;367(5):423-34.

4. Centers for Disease Control and Prevention. Preexposure prophylaxis for the prevention of HIV infection in the United States, -2014: A Clinical Practice Guideline. 2014.

5. Sullivan PS, Smith DK, Mera-Giler R, Siddiqi A, Gunnels B, Harris N, et al. The impact of pre-exposure prophylaxis with TDF/FTC on HIV diagnoses, 2012-2016, United States. 22nd International AIDS Conference; Amsterdam, Netherlands2018.

6. Buchbinder SP, Liu AY. CROI 2019: Advances in HIV prevention and plans to end the epidemic. Top Antivir Med. 2019;27(1):8-25.

7. Smith DK, Van Handel M, Grey J. Estimates of adults with indications for HIV pre-exposure prophylaxis by jurisdiction, transmission risk group, and race/ethnicity, United States, 2015. Annl Epidemiol. 2018.

8. Sullivan PS, Giler RM, Mouhanna F, Pembleton ES, Guest JL, Jones J, et al. Trends in the use of oral emtricitabine/tenofovir disoproxil fumarate for pre-exposure prophylaxis against HIV infection, United States, 2012-2017. Ann Epidemiol. 2018;28(12):833-40.

9. Hurt CB. PrEParing providers: the next challenge in implementing human immunodeficiency virus preexposure prophylaxis. Sex Transm Dis. 2018;45(7):459-61. 
10. Bacon O, Gonzalez R, Andrew E, Potter MB, Iniguez JR, Cohen SE, et al. Informing strategies to build PrEP capacity among San Francisco Bay Area clinicians. J Acquir Immune Defic Syndr. 2017;74(2):175-9.

11. Hart-Cooper GD, Allen I, Irwin CE, Jr., Scott H. Adolescent health providers' willingness to prescribe pre-eposure Prophylaxis (PrEP) to youth at risk of HIV infection in the United States. J Adolesc Health. 2018;63(2):242-4.

12. Walsh JL, Petroll AE. Factors related to pre-exposure prophylaxis prescription by U.S. primary care physicians. Am J Prev Med. 2017;52(6):e165-e72.

13. Wood BR, McMahan VM, Naismith K, Stockton JB, Delaney LA, Stekler JD. Knowledge, practices, and barriers to HIV preexposure prophylaxis prescribing among Washington State medical providers. Sex Transm Dis. 2018;45(7):452-8.

14. Petroll AE, Walsh JL, Owczarzak JL, McAuliffe TL, Bogart LM, Kelly JA. PrEP awareness, familiarity, comfort, and prescribing experience among US primary care providers and HIV specialists. AIDS Behav. 2017;21(5):1256-67.

15. Blackstock OJ, Moore BA, Berkenblit GV, Calabrese SK, Cunningham CO, Fiellin DA, et al. a crosssectional online survey of HIV pre-exposure prophylaxis adoption among primary care physicians. J Gen Intern Med. 2017;32(1):62-70.

16. Zablotska IB, O'Connor CC. Preexposure prophylaxis of HIV infection: the role of clinical practices in ending the HIV epidemic. Curr HIV/AIDS Rep. 2017;14(6):201-10.

17. Desai M, Gafos M, Dolling D, McCormack S, Nardone A. Healthcare providers' knowledge of, attitudes to and practice of pre-exposure prophylaxis for HIV infection. HIV Med. 2016;17(2):133-42.

18. Conniff J, Evensen A. Preexposure prophylaxis (PrEP) for HIV prevention: the primary care perspective. J Am Board Fam Med. 2016;29(1):143-51.

19. Krakower DS, Mayer KH. The role of healthcare providers in the roll out of preexposure prophylaxis. Curr Opin HIV AIDS. 2016;11(1):41-8.

20. Mullins TLK, Zimet G, Lally M, Xu J, Thornton S, Kahn JA. HIV care providers' intentions to prescribe and actual prescription of pre-exposure prophylaxis to at-risk adolescents and adults. AIDS Patient Care STDS. 2017;31(12):504-16.

21. Blumenthal J, Jain S, Krakower D, Sun X, Young J, Mayer K, et al. Knowledge is power! Increased provider knowledge scores regarding pre-exposure prophylaxis (PrEP) are associated with higher rates of PrEP prescription and future intent to prescribe PrEP. AIDS Behav. 2015;19(5):802-10.

22. Krakower DS, Mayer KH. Pre-exposure prophylaxis to prevent HIV infection: current status, future opportunities and challenges. Drugs. 2015;75(3):243-51.

23. Calabrese SK, Earnshaw VA, Underhill K, Hansen NB, Dovidio JF. The impact of patient race on clinical decisions related to prescribing HIV pre-exposure prophylaxis (PrEP): assumptions about sexual risk compensation and implications for access. AIDS Behav. 2014;18(2):226-40.

24. Calabrese SK, Earnshaw VA, Krakower DS, Underhill K, Vincent W, Magnus M, et al. A closer look at racism and heterosexism in medical students' clinical decision-making related to HIV pre-exposure 
prophylaxis (prep): implications for PrEP education. AIDS Behav. 2018;22(4):1122-38.

25. Calabrese SK, Earnshaw VA, Underhill K, Krakower DS, Magnus M, Hansen NB, et al. Prevention paradox: medical students are less inclined to prescribe HIV pre-exposure prophylaxis for patients in highest need. J Int AIDS Soc. 2018;21(6):e25147.

26. Armstrong M, Nigh E, Gill-WiehI G, Doblecki-Lewis S. Medical students' perspectives on HIV prevention education in the preclinical years. AIDS Res Hum Retroviruses. 2018;34(10):817-9.

27. Eweka I, Fleuriscar J, Fleuriscar J, Adeyemi-Jones A, Dillon B, Albritton T. Medical students reflect on the future of pre-exposure prophylaxis use among adolescents and young adults. Prev Med. 2018;113:122-3.

28. Nunn AS, Brinkley-Rubinstein L, Oldenburg CE, Mayer KH, Mimiaga M, Patel R, et al. Defining the HIV pre-exposure prophylaxis care continuum. AIDS. 2017;31(5):731-4.

29. Farmer EK, Koren DE, Cha A, Grossman K, Cates DW. The Pharmacist's expanding role in HIV preexposure prophylaxis. AIDS Patient Care STDS. 2019;33(5):207-13.

30. Saberi P, Dong BJ, Johnson MO, Greenblatt RM, Cocohoba JM. The impact of HIV clinical pharmacists on HIV treatment outcomes: a systematic review. Patient Prefer Adherence. 2012;6:297322.

31. Adams JL, Shelley K, Nicol MR. Review of real-world implementation data on emtricitabine-tenofovir disoproxil fumarate as HIV pre-exposure prophylaxis in the United States. Pharmacotherapy. 2019;39(4):486-500.

32. Unni EJ, Lian N, Kuykendall W. Understanding community pharmacist perceptions and knowledge about HIV preexposure prophylaxis (PrEP) therapy in a mountain west state. J Am Pharm Assoc (2003). 2016;56(5):527-32.e1.

33. Nelson LE, McMahon JM, Leblanc NM, Braksmajer A, Crean HF, Smith K, et al. Advancing the case for nurse practitioner-based models to accelerate scale-up of HIV pre-exposure prophylaxis. J Clin Nurs. 2019;28(1-2):351-61.

34. Castel AD, Feaster DJ, Tang W, Willis S, Jordan H, Villamizar K, et al. Understanding HIV care provider attitudes regarding intentions to prescribe PrEP. J Acquir Immune Defic Syndr. 2015;70(5):520-8.

35. Associated Medical Schools of New York. Medical school student data, 2018. Available from: https://amsny.org/initiatives/medical-education/student-data/. Accessed 20 May 2019.

36. New York State Education Department. New York State nursing programs: doctoral degree programs, 2014. Available from: http://www.op.nysed.gov/prof/nurse/nurseprogs-doctoral.htm. Accessed 20 May 2019.

37. Accredidation Council for Pharmacy Education. Programs by state. Aailable from: https://www.acpeaccredit.org/accredited-programs-by-state/. Accessed 20 May 2019.

38. New York State Education Department. License statistics: nurse practitioner, 2019. Available from: http://www.op.nysed.gov/prof/nurse/nursecounts.htm. Accessed 20 May 2019. 
39. New York State Education Department. License statistics: pharmacy, 2019. Available from: http://www.op.nysed.gov/prof/pharm/pharmcounts.htm. Accessed 20 May 2019.

40. Wu H, Mendoza MC, Huang YA, Hayes T, Smith DK, Hoover KW. Uptake of HIV preexposure prophylaxis among commercially insured persons-united states, 2010-2014. Clin Infect Dis. 2017;64(2):144-9.

41. Silapaswan A, Krakower D, Mayer KH. Pre-exposure prophylaxis: a narrative review of provider behavior and interventions to increase PrEP implementation in primary care. J Gen Intern Med. 2016;32(2):192-8.

42. Bauler TJ, Cole S, Gibb T, Van Enk R, Lutwick L, Dickinson BL. HIV/AIDS: a case-based learning module for first-year medical students. J Microbiol Biol Educ. 2018;19(2).

43. Nori P, Madaline T, Munjal I, Bhar S, Guo Y, Seo SK, et al. Developing interactive antimicrobial stewardship and infection prevention curricula for diverse learners: a tailored approach. Open Forum Infect Dis. 2017;4(3):ofx117.

\section{Tables}

Table 1. Demographic and clinical characteristics based on health professional student type

\begin{tabular}{|c|c|c|c|c|}
\hline & $\begin{array}{l}\text { Full Sample } \\
\text { (Total N = 744) }\end{array}$ & $\begin{array}{l}\text { MD Students } \\
\text { (Total } \mathbf{N}=376 \text { ) }\end{array}$ & $\begin{array}{l}\text { NP Students } \\
\text { (Total } N=79 \text { ) }\end{array}$ & $\begin{array}{l}\text { PharmD Students } \\
\text { (Total N = 289) }\end{array}$ \\
\hline Variable & $\mathrm{M}(\mathrm{SD})$ or $\%(\mathrm{~N})$ & $\mathrm{M}(\mathrm{SD})$ or $\%(\mathrm{~N})$ & $\mathrm{M}(\mathrm{SD})$ or $\%(\mathrm{~N})$ & $\mathrm{M}(\mathrm{SD})$ or $\%(\mathrm{~N})$ \\
\hline Age & $25.10(4.25)$ & $25.38(2.81)$ & $31.46(7.45)$ & $23.00(2.37)$ \\
\hline \multicolumn{5}{|l|}{ Sex } \\
\hline Male & $38.68 \%(287)$ & $44.00 \%(165)$ & $22.78 \%(18)$ & $36.11 \%(104)$ \\
\hline Female & $61.19 \%(454)$ & $55.73 \%(209)$ & $77.22 \%(61)$ & $63.89 \%(184)$ \\
\hline Nonbinary & $0.13 \%(1)$ & $0.27 \%(1)$ & $0.00 \%(0)$ & $0.00 \%(0)$ \\
\hline \multicolumn{5}{|l|}{ Race } \\
\hline Black/African American & $4.12(30)$ & $4.66 \%(17)$ & $3.80 \%(3)$ & $3.52 \%(10)$ \\
\hline Asian & $24.18 \%(176)$ & $19.18 \%(70)$ & $6.33 \%(5)$ & $35.56 \%(101)$ \\
\hline White & $65.80 \%(479)$ & $67.67 \%(247)$ & $84.81 \%(67)$ & $58.10 \%(165)$ \\
\hline Other & $5.91 \%(43)$ & $8.49 \%(31)$ & $5.06 \%(4)$ & $2.82 \%(8)$ \\
\hline \multicolumn{5}{|l|}{ Class year } \\
\hline $1^{\text {st }}$ year & $25.14 \%(186)$ & $25.40 \%(95)$ & $27.85 \%(22)$ & $24.04 \%(69)$ \\
\hline $2^{\text {nd }}$ year & $23.51 \%(174)$ & $24.60 \%(91)$ & $21.52 \%(17)$ & $23.00 \%(66)$ \\
\hline $3^{\text {rd }}$ year & $24.59 \%(182)$ & $25.13 \%(94)$ & $26.58 \%(21)$ & $23.34 \%(67)$ \\
\hline $4^{\text {th }} / 5^{\text {th }}$ year & $26.89 \%(198)$ & $25.13 \%(94)$ & $24.05 \%(19)$ & $29.62 \%(85)$ \\
\hline \multicolumn{5}{|c|}{ Experience with HIV-infected populations } \\
\hline Yes & $61.02 \%(454)$ & $69.95 \%(263)$ & $92.41 \%(73)$ & $40.83 \%(118)$ \\
\hline No & $38.98 \%(290)$ & $30.05 \%(113)$ & $7.59 \%(6)$ & $59.17 \%(171)$ \\
\hline \multicolumn{5}{|c|}{ Know someone who has used PrEP } \\
\hline Yes & $27.47 \%(203)$ & $28.43 \%(106)$ & $26.58 \%(21)$ & $26.48 \%(76)$ \\
\hline No & $72.53 \%(536)$ & $71.58 \%(267)$ & $73.42 \%(58)$ & $73.52 \%(211)$ \\
\hline
\end{tabular}

Note. Due to some participants choosing not to respond to certain questions, numbers may not add up to the total. 
Table 2. Differences in PrEP awareness, knowledge, familiarity with prescribing guidelines, and comfort performing PrEP-related tasks

\begin{tabular}{lllll}
\hline & $\begin{array}{l}\text { MD } \\
\text { Students }\end{array}$ & $\begin{array}{l}\text { NP } \\
\text { students }\end{array}$ & $\begin{array}{l}\text { PharmD } \\
\text { students }\end{array}$ & ANOVA \\
\cline { 2 - 5 } & $\mathrm{M}(\mathrm{SD})$ & $\mathrm{M}(\mathrm{SD})$ & $\mathrm{M}(\mathrm{SD})$ & $\mathrm{F}(\mathrm{df})$ \\
\hline PrEP awareness & $0.86(0.35)$ & $\begin{array}{l}0.78_{\mathrm{a}} \\
(0.35)\end{array}$ & $0.91_{\mathrm{a}}(0.29)$ & $4.42(2,741)^{*}$ \\
& & & & \\
\hline PrEP knowledge & $1.38_{\mathrm{a}}$ & $1.53_{\mathrm{b}}$ & $1.93_{\mathrm{a}, \mathrm{b}}$ & 11.03 \\
& $(1.39)$ & $(1.58)$ & $(1.62)$ & $(2,738)^{* * *}$ \\
\hline Familiarity with PrEP prescription guidelines & $2.51_{\mathrm{a}}$ & $2.25_{\mathrm{b}}$ & $3.33_{\mathrm{a}, \mathrm{b}}$ & 41.61 \\
& $(1.31)$ & $(1.36)$ & $(1.24)$ & $(2,739)^{* * *}$ \\
\hline $\begin{array}{l}\text { Comfort performing PrEP-related clinical } \\
\text { activities }\end{array}$ & $4.05_{\mathrm{a}}$ & $3.86(0.98)$ & $3.88(0.92)_{\mathrm{a}}$ & $3.64(2,739)^{*}$ \\
\hline
\end{tabular}

Note. Values with the same subscripts differ significantly at $\mathrm{p}<.05 .{ }^{*} p<.05 .{ }^{* * *} p<.001$

Table 3. Participant characteristics associated with willingness to prescribe PrEP

\begin{tabular}{llll}
\hline Variable & $\boldsymbol{b}$ & $\boldsymbol{S E}$ & $\boldsymbol{t}$ \\
\hline Intercept & 4.44 & 0.15 & $29.07^{* * *}$ \\
\hline Student type $(\mathrm{MD}=1, \mathrm{NP}=0)$ & 0.19 & 0.10 & $1.88^{\dagger}$ \\
\hline Sex (female $=1$, male $=0$ ) & 0.00 & 0.00 & -0.31 \\
\hline Race (white $=1$, non-white $=0)$ & -0.11 & 0.09 & -1.22 \\
\hline Program year (1-4/5) & -0.10 & 0.04 & $-2.88^{* *}$ \\
\hline Familiarity with PrEP prescription guidelines & 0.09 & 0.03 & $2.98^{* *}$ \\
\hline PrEP knowledge & 0.02 & 0.03 & 0.67 \\
\hline $\begin{array}{l}\text { Know someone who has used PrEP } \\
\text { (yes }=1, \text { no }=0 \text { ) }\end{array}$ & 0.15 & 0.09 & 1.61 \\
\hline
\end{tabular}

Note. ${ }^{\dagger} p<.10 .{ }^{* *} p<.01 .{ }^{* * *} p<.001$.

Table 4. Participant characteristics associated with willingness to refer a PrEP candidate to another provider 


\begin{tabular}{llll}
\hline Variable & \multicolumn{1}{l}{$\boldsymbol{b}$} & $\boldsymbol{S} \boldsymbol{E}$ & $\boldsymbol{t}$ \\
\hline Intercept & 4.57 & 0.09 & $52.84^{* * *}$ \\
Student type $(\mathrm{MD}=1, \mathrm{PharmD}=0)$ & 0.11 & 0.05 & $2.09^{*}$ \\
\hline Student type $(\mathrm{NP}=1$, PharmD =0) & 0.05 & 0.08 & 0.58 \\
\hline Sex (female $=1$, male $=0)$ & 0.00 & 0.00 & -0.42 \\
\hline Race (white $=1$, non-white $=0)$ & 0.04 & 0.05 & 0.89 \\
\hline Program year $(1-4 / 5)$ & 0.02 & 0.02 & 0.74 \\
\hline Familiarity with PrEP prescription guidelines & 0.02 & 0.02 & 1.17 \\
\hline PrEP knowledge & 0.02 & 0.02 & 1.03 \\
\hline Know someone who has used PrEP & -0.03 & 0.05 & -0.47 \\
\hline
\end{tabular}

Note. ${ }^{*} p<.05 .{ }^{* * *} p<.001$. 